Surface Review and Letters, Vol. 15, No. 5 (2008) 599-603

(c) World Scientific Publishing Company

\title{
SIZE-DEPENDENT ELASTIC MODULUS AND FRACTURE TOUGHNESS OF THE NANOFILM WITH SURFACE EFFECTS
}

\author{
JIAN-GANG GUO*, LI-JUN ZHOU ${ }^{\dagger}$ and YA-PU ZHAO ${ }^{\ddagger}$ \\ *Department of Mechanics, Tianjin University, \\ Tianjin 300072, China \\ ${ }^{\dagger}$ Department of Mechanical Engineering, \\ Tianjin University of Technology and Education, \\ Tianjin 300222, China \\ ${ }_{\ddagger}^{\ddagger}$ State Key Laboratory of Nonlinear Mechanics, \\ Institute of Mechanics, Chinese Academy of Sciences, \\ Beijing 100190, China \\ yzhao@imech.ac.cn
}

Received 23 January 2008

\begin{abstract}
The effective elastic modulus and fracture toughness of the nanofilm were derived with the surface relaxation and the surface energy taken into consideration by means of the interatomic potential of an ideal crystal. The size effects of the effective elastic modulus and fracture toughness were discussed when the thickness of the nanofilm was reduced. And the dependence of the size effects on the surface relaxation and surface energy was also analyzed.
\end{abstract}

Keywords: Size-dependent; surface effects; elastic modulus; fracture toughness; nanofilm.

PACS Number(s): 62.20.Dc, 68.35.-p, 68.60.-p, 68.60.Bs

\section{Introduction}

The elastic modulus and fracture toughness at nanoscale are fundamental mechanical properties for nano-devices and nano-manufacturing. For example, in the recent fabrication of a full-functional nanotube radio by Zettl's group, ${ }^{1}$ resonant frequency of a carbon nanotube (CNT) was utilized to fabricate the tuner of the nanoradio, and the resonant frequency of the CNT is proportional to the square-root of the Young's modulus of the CNT. Therefore, whether the elastic modulus of CNT exhibits scale-dependent will be of big concern for the design and fabrication of nanotube radio. The fracture properties of
$\mathrm{ZnO}$ nanowires and nanobelts were also concerned in the field of the nanopiezotronics, such as nanogenerator, etc. ${ }^{2}$ The size-dependent Young's modulus and fracture toughness were considered two of the four research opportunities of nanomechanics, ${ }^{3}$ and there is a big driving force for the nanofracture mechanics in the practical application in nanotechnology. ${ }^{4}$

As a matter of fact, the Young's modulus of a nanostructure has been proved to be size-dependent by lots of experiment results ${ }^{5-13}$ and theoretical researches. ${ }^{14-24}$ The studies showed that the Young's modulus was stiffened ${ }^{5-8}$ or softened ${ }^{9-13}$ with the decrease of the size, and most of researchers believed

${ }_{\ddagger}^{\ddagger}$ Corresponding author. 
that the size effects should be caused by the influence of the surface effects. Guo and Zhao ${ }^{22,23}$ built a three-dimensional simple cubic crystal model of a nanofilm with the surface relaxation and the surface energy taken into consideration, and derived the elastic moduli of the nanofilm, and then proved the elastic moduli to be size- and surface-dependent. They also studied the bending elastic properties of a one-dimensional nanostructure, and derived the sizedependent effective bending modulus and effective flexural rigidity of a nanobeam. ${ }^{24}$

In engineering application, besides the Young's modulus and flexural rigidity, the bulk modulus is also an important mechanical property. But until now, there are few researches on the size-dependent bulk modulus of a one-dimensional nanostructure. The dependence of the bulk modulus of nanocrystalline nickel on the grain size was investigated by means of molecular dynamics simulations. ${ }^{25}$ And the calculated data showed an increasing compressibility with decreasing grain size and can be modeled by a rule of mixture.

In this paper, with the surface relaxation and the surface energy taken into consideration, the sizedependent bulk modulus of a nanofilm will be derived by means of the interatomic potential of an ideal crystal. And the Young's modulus and shear modulus of it will also be discussed if the Poisson's ratio can be assumed to be a constant. In addition, according to the classical Griffith's theory of linear elastic fracture mechanics, the fracture toughness of brittle materials is the function of Young's modulus and surface energy. So we will also give a simple discussion on the size-dependent fracture toughness of a brittle nanofilm.

\section{The Effective Bulk Modulus of a Nanofilm}

According to the theory of solid-state physics, the interatomic potential of an ideal crystal can be expressed as:

$$
u(r)=\frac{p q}{p-q} \varepsilon_{b}\left[\frac{1}{p}\left(\frac{a_{0}}{r}\right)^{p}-\frac{1}{q}\left(\frac{a_{0}}{r}\right)^{q}\right],
$$

where $p$ and $q$ dependent on the shapes of potential curves, $a_{0}$ is the interatomic distance in equilibrium, and $\varepsilon_{b}$ is the minimum value of the potential.
When the values of $p$ and $q$ are specified as $p=$ 12 and $q=6$, the potential function becomes the classical Lennard-Jones (L-J) potential

$$
u(r)=\varepsilon_{b}\left[\left(\frac{a_{0}}{r}\right)^{12}-2\left(\frac{a_{0}}{r}\right)^{6}\right] .
$$

In equilibrium state, there exists

$$
\left.\frac{d u(r)}{d r}\right|_{r=a_{0}}=0
$$

and

$$
\left.\frac{d^{2} u(r)}{d r^{2}}\right|_{r=a_{0}}=\frac{72}{a_{0}^{2}} \varepsilon_{b} .
$$

If the atomic number of the ideal crystal with the volume $V$ is specified to be $N_{0}$, then the internal energy of the crystal can be written as

$$
U(r)=\frac{1}{2} N_{0} n_{\mathrm{c}} u(r),
$$

where $n_{\mathrm{c}}$ is the atomic coordination number, and the volume $V$ can be calculated by

$$
V=N_{0} r^{3} .
$$

According to the theory of solid-state physics, the bulk modulus of a crystal can be written as

$$
\begin{aligned}
K & =V\left(\frac{d^{2} U}{d V^{2}}\right) \\
& =V\left[\frac{d^{2} U}{d r^{2}} \cdot\left(\frac{d r}{d V}\right)^{2}+\frac{d U}{d r} \cdot \frac{d^{2} r}{d V^{2}}\right] .
\end{aligned}
$$

Substituting Eqs. (2)-(5) in Eq. (7), the bulk modulus of the crystal can be derived as

$$
K=\frac{4 n_{\mathrm{c}} \varepsilon_{b}}{a_{0}^{3}} .
$$

In Eq. (8), the value of the bulk modulus is dependent on the interatomic distance in equilibrium $a_{0}$ and the minimum value of the potential $\varepsilon_{b}$. For a macroscopic ideal crystal, the values of $\varepsilon_{b}$ and $a_{0}$ are fixed, so the bulk modulus is a constant. However, for a nanostructural crystal, due to the influence of the surface effects, the values of $a_{0}$ and $\varepsilon_{b}$ will not be fixed for a certain material, and correspondingly the bulk modulus is not a constant. At the nanoscale, the surface effects have great influence on the physical properties of structures due to the large ratio of surface to volume. The surface effects, such as 
surface relaxation, surface reconstruction and surface energy, etc., can change the values of $a_{0}$ and $\varepsilon_{b}$, and make $a_{0}$ and $\varepsilon_{b}$ be size- and surface-dependent. Taking the influence of the surface relaxation and the surface energy into consideration, we define the new interatomic distance of a nanofilm in equilibrium and the minimum value of the potential as $a^{*}$ and $\varepsilon^{*}$, respectively. And the bulk modulus of the nanofilm is assumed to have the form of Eq. (8). Then the dimensionless bulk modulus of the nanofilm can be written as

$$
\bar{K}=\frac{K_{\text {film }}}{K}=\left(\frac{a_{0}}{a^{*}}\right)^{3} \cdot\left(\frac{\varepsilon^{*}}{\varepsilon_{b}}\right) .
$$

In the following, the size- and surface-dependent expressions of $a^{*}$ and $\varepsilon^{*}$ will be derived, respectively. Firstly, the relaxation coefficient $k$ is introduced. On the free surface of the nanofilm, several layers of atoms will relax due to the imperfection of the coordination numbers $(\mathrm{CNs})$. As a result, the interatomic distance in equilibrium will change. The relaxation coefficient $k$ is a parameter scaling the variation, which is the ratio of the relaxed interatomic distance and the unrelaxed interatomic distance, i.e., $a=k a_{0}$. Apparently, the relaxation may be in contraction when $k_{i}<1$, and be in expansion when $k_{i}>1$. We define an average value of the interatomic distances as the value of the interatomic distances in equilibrium $a^{*}$ when the surface relaxation happens, i.e.,

$$
a^{*}=\frac{(N+2 n k) a_{0}}{N+2 n},
$$

where $N$ is the number of the unrelaxed atomic layers, and $n$ is the number of the relaxed atomic layers. Furthermore, the dimensionless form of the distance can be written as

$$
\delta=\frac{a_{0}}{a^{*}}=\frac{N+2 n}{N+2 n k} .
$$

According to Eq. (5), the potential of the ideal crystal in equilibrium state can be written as

$$
U=\frac{1}{2} N_{0} n_{\mathrm{c}} \varepsilon_{b} .
$$

While the potential of the nanofilm in equilibrium state is

$$
\begin{aligned}
U_{f} & =\frac{1}{2}\left[\left(n_{\mathrm{c}}-m\right) \eta+n_{\mathrm{c}}(1-\eta)\right] N_{0} \varepsilon^{*} \\
& =\frac{1}{2} N_{0}\left(n_{\mathrm{c}}-m \eta\right) \varepsilon^{*}
\end{aligned}
$$

where the parameter $m$ denotes the decrease of atomic coordination number on the free surface of the nanofilm, and $\eta$ denotes the ratio of surface atom numbers and total atom numbers, which can be expressed as

$$
\eta=\frac{2 n}{N+2 n} .
$$

If we split an ideal crystal into a nanofilm with two free surfaces, then there is the following potential relationship, $U-U_{f}=\gamma A$. Thus, we can further obtained

$$
\frac{\varepsilon^{*}}{\varepsilon_{b}}=\frac{n_{\mathrm{c}}}{n_{\mathrm{c}}-m \eta} \cdot\left(1-\frac{\gamma A}{U}\right),
$$

where the area can be calculated by $A=N_{0} \eta a_{0}^{2}$.

Substituting Eqs. (11) and (15) in Eq. (9), we can derive the dimensionless effective bulk modulus of the nanofilm

$$
\begin{aligned}
\bar{K} & =\left(\frac{a_{0}}{a^{*}}\right)^{3} \cdot\left(\frac{\varepsilon^{*}}{\varepsilon_{b}}\right) \\
& =\delta^{3} \cdot \frac{n_{\mathrm{c}}}{n_{\mathrm{c}}-m \eta} \cdot\left(1-\frac{2 \eta}{n_{\mathrm{c}}} \cdot \frac{\gamma a_{0}^{2}}{\varepsilon_{b}}\right) .
\end{aligned}
$$

Furthermore, it can be written as

$$
\bar{K}=\beta \cdot\left(1-\frac{\gamma a_{0}^{2}}{\alpha \varepsilon_{b}}\right)
$$

where

$$
\xi=\frac{n_{\mathrm{c}}-m \eta}{n_{\mathrm{c}}}, \quad \beta=\frac{\delta^{3}}{\xi}, \quad \alpha=\frac{n_{\mathrm{c}}}{2 \eta} .
$$

\section{Young's Modulus, Shear Modulus, and Fracture Toughness}

In the section above, the dimensionless effective bulk modulus of a nanofilm was derived. For isotropic materials, there are the following relations among Young's modulus, shear modulus, and bulk modulus:

$$
E=3 K(1-2 \nu), \quad G=\frac{3 K(1-2 \nu)}{2(1+\nu)},
$$

where $E$ is Young's modulus; $G$, the shear modulus, and $\nu$ is the Poisson's ratio. Poisson's ratio is usually assumed to vary slightly. So the effective Young's modulus and shear modulus of the nanofilm can be derived, and the dimensionless forms of them 
are, respectively,

$$
\begin{aligned}
& \bar{E}_{\text {eff }}=\frac{E_{\text {film }}^{\text {eff }}}{K}=3(1-2 \nu) \bar{K}, \\
& \bar{G}_{\text {eff }}=\frac{G_{\text {filn }}^{\text {eff }}}{K}=\frac{3(1-2 \nu)}{2(1+\nu)} \bar{K} .
\end{aligned}
$$

In addition, according to the Griffith's theory of linear elastic fracture mechanics, the fracture toughness of brittle materials is

$$
K_{C}=\sqrt{\pi E \gamma},
$$

which is dependent on Young's modulus $E$ and surface energy $\gamma$.

If the equation can be applied to nanostructures, then the dimensionless effective fracture toughness of the nanofilm can be derived when the surface energy $\gamma$ is assumed to be constant,

$$
\bar{K}_{C}=\frac{K_{C}^{*}}{K_{C}}=\left(\frac{E_{\mathrm{film}}^{\mathrm{eff}}}{E}\right)^{\frac{1}{2}}=\sqrt{\bar{K}}
$$

where $K_{C}$ is the macroscopic fracture toughness of the materials, and $K_{C}^{*}$ the effective fracture toughness of the nanofilm, which is in proportion to the square root of the dimensionless effective bulk modulus.

\section{Discussion and Conclusion}

An example is given to discuss the size- and surfacedependence of elastic moduli and fracture toughness. The values of parameters for calculation are specified in Table 1. The surface relaxation usually exists at the several layers of atoms, and the degree of the relaxation decays greatly along the inner-normal direction of the free surface, and no $\mathrm{CN}$ reduction is expected for $n>3$. So, for the sake of simplification and without loss of generality, we assume that the relaxation of the crystal only occurs on the outmost atomic layer, i.e., $n=1$.

Table 1. The values of parameters in the example.

\begin{tabular}{cc}
\hline Symbol & Value \\
\hline$a_{0}$ & $0.2556 \times 10^{-10} \mathrm{~m}$ \\
$n_{\mathrm{c}}$ & 12 \\
$m$ & 6 \\
$\varepsilon$ & $0.69 \times 10^{-19} \mathrm{~J}$ \\
$\gamma$ & $1.952 \mathrm{~N} \cdot \mathrm{m}^{-1}$ \\
$\nu$ & 0.3 \\
\hline
\end{tabular}

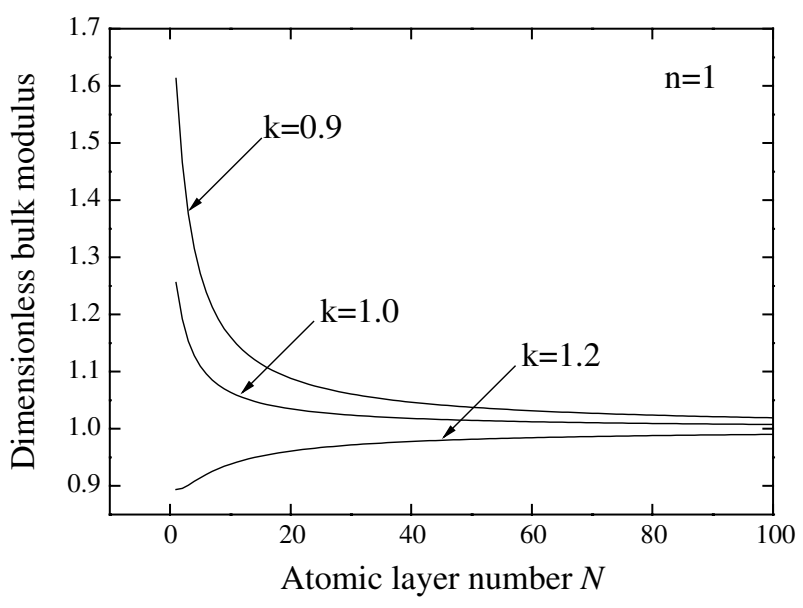

Fig. 1. The variations of dimensionless bulk modulus with the decrease of atomic layer number.

Figure 1 shows the variations of the dimensionless effective bulk modulus of the nanofilm with the decrease of atomic layer number. It can be seen from Fig. 1 that the dimensionless effective bulk modulus varies with the decreasing the thickness of the nanofilm when the number of the atomic layer is less than about 60 . And the thinner the nanofilm is, the sharper the curve becomes. In addition, the variations of the effective bulk modulus are also dependent on the surface effects. For the nanofilm with the same thickness, the smaller the surface relaxation is, the greater the value of the effective bulk modulus is.

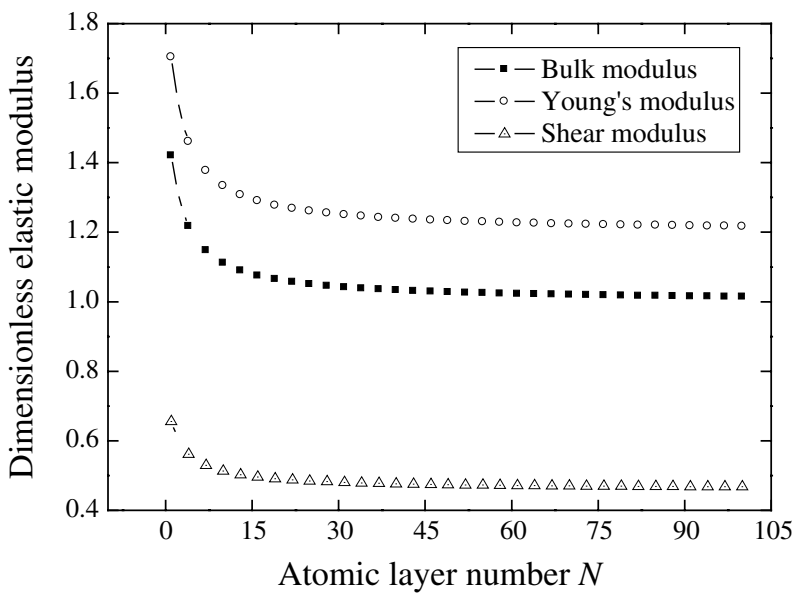

Fig. 2. The variations of dimensionless bulk modulus, Young's modulus, and shear modulus with the decrease of atomic layer number when the surface relaxation coefficient $k=1$. 
For the case of $k=1$, the bulk modulus is still enhanced due to the influence of the positive surface energy.

The effective Young's modulus, shear modulus, and fracture toughness have the same variations with the effective bulk modulus when the thickness of the nanofilm is reduced. Figure 2 shows the variations of the effective Young's modulus, shear modulus, and bulk modulus when the relaxation coefficient $k$ equals to one, i.e., $k=1$. The effective fracture toughness of a nanofilm is in proportion to the square root of the dimensionless effective bulk modulus.

\section{Acknowledgments}

This work was supported by the National Basic Research Program of China (973 Program, Grant no. 2007CB310500), National High-tech R\&D Program of China (863 Program, Grant no. 2007AA04Z348), and National Natural Science Foundation of China (NSFC, Grant nos. 10772180 and 10721202).

\section{References}

1. K. Jensen, J. Weldon, H. Garcia and A. Zettl, Nano Lett. 7 (2007) 3508.

2. Z. L. Wang, Nano Lett. 10 (2007) 20.

3. W. W. Gerberich, W. M. Mook, M. J. Cordill, J. M. Jungk, B. Boyce, T. Friedmann, N. R. Moody and D. Yang, Int. J. Fract. 138 (2006) 75.

4. Z. W. Wang, K. Finkelstein, K. C. Ma and Z. L. Wang, Appl. Phys. Lett. 90 (2007) 113115.

5. S. Cuenot, S. Demoustier-Champagne and B. Nysten, Phys. Rev. Lett. 85 (2000) 1690.

6. C. Q. Chen, Y. Shi, Y. S. Zhang, J. Zhu and Y. J. Yan, Phys. Rev. Lett. 96 (2006) 075505.
7. M. K. Shin, S. I. Kim, S. J. Kim, S. K. Kim, H. Lee and G. M. Spinks, Appl. Phys. Lett. 89 (2006) 231929

8. E. P. S. Tan, Y. Zhu, T. Yu, L. Dai, C. H. Sow, V. B. C. Tan and C. T. Lim, Appl. Phys. Lett. 90 (2007) 163112.

9. P. Villain, P. Goudeau, P.-O. Renault and K. F. Badwi, Appl. Phys. Lett. 81 (2002) 4365.

10. M. C. Salvadori, I. G. Brown, A. R. Vaz, L. L. Melo and M. Cattani, Phys. Rev. B 67 (2003) 153404.

11. S. G. Nilsson, X. Borrisé and L. Montelius, Appl. Phys. Lett. 85 (2004) 3555.

12. M. Kopycinska-Muller, R. H. Geiss, J. Muller and D. C. Hurley, Nanotechnology 16 (2005) 703.

13. Q. H. Xiong, N. Duarte, S. Tadigadapa and P. C. Eklund, Nano Lett. 6 (2006) 1904.

14. F. H. Streitz, R. C. Cammarata and K. Sieradzki, Phys. Rev. B 49 (1994) 10699.

15. V. B. Shenoy, Phys. Rev. B 71 (2005) 094104.

16. L. G. Zhou and H. C. Huang, Appl. Phys. Lett. 84 (2004) 1940 .

17. D. Wolf, Appl. Phys. Lett. 58 (1991) 2081.

18. Z. Y. Yang and Y. P. Zhao, Surf. Rev. Lett. 14 (2007) 661.

19. P. Villain, P. Beauchamp, K. F. Badwi, P. Goudeau and P. O. Renault, Scripta Mater. 50 (2004) 1247.

20. M. X. Gu, C. Q. Sun, Z. Chen, T. C. A. Yeung, S. Li, C. M. Tan and V. Nosik, Phys. Rev. B 75 (2007) 125403.

21. G. Ouyang, X. Tan and G. Yang, Phys. Rev. B 74 (2006) 195408.

22. J. G. Guo and Y. P. Zhao, J. Appl. Phys. 98 (2005) 074306.

23. J. G. Guo and Y. P. Zhao, Surf. Rev. Lett. 14 (2007) 667.

24. J. G. Guo and Y. P. Zhao, Nanotechnology 98 (2007) 074306.

25. S. J. Zhao, K. Albe and H. Hahn, Scripta Mater. 55 (2006) 473. 